\title{
The Holomorphic Anomaly of Topological Strings
}

\author{
Carlo Becchi, Stefano Giusto ${ }^{1}$, Camillo Imbimbo ${ }^{2}$ \\ ${ }^{1}$ Dipartimento di Fisica dell'Università di Genova \\ Via Dodecaneso 33, I-16146 Genova \\ ${ }^{2}$ I.N.F.N. Sezione di Genova \\ Via Dodecaneso 33, I-16146 Genova
}

\begin{abstract}
We show that the BRS operator of the topological string B model is not holomorphic in the complex structure of the target space. This implies that the so-called holomorphic anomaly of topological strings should not be interpreted as a BRS anomaly.
\end{abstract}

\section{Introduction and Conclusions}

There exist two topological sigma models whose target space is a Calabi-Yau complex three-fold $X$ [1]: The A-model, which depends on the complexified Kähler structures of $X$ and the B-model, which depends on its complex structures. The moduli of both the $\mathrm{A}$ and the $\mathrm{B}$ model are in correspondence with integrated two-forms which are closed under the BRS operator characterizing the topological theory. The moduli spaces of both the A and the B model carry a natural complex structure; The operators corresponding to the holomorphic moduli are non-trivial elements of the BRS cohomology, while those associated to the anti-holomorphic ones are BRS-exact.

This is understood in terms of the underlying $N=2$ superconformal model [2]: (Anti)holomorphic deformations correspond to marginal operators $O_{\alpha}^{(2)}=\tilde{Q} \bar{Q} \Phi_{\alpha}\left(O_{\bar{\alpha}}^{(2)}=Q \bar{Q} \Phi_{\bar{\alpha}}\right)$, where $Q \tilde{Q}$ are the two left-handed supersymmetry charges, and $\bar{Q} \bar{Q}$ are the right-handed ones. $\Phi_{\alpha}\left(\Phi_{\bar{\alpha}}\right)$ are (anti)chiral superfields. Upon twisting, $Q+\bar{Q} \equiv s$ is identified with the BRS operator of the topological model: Thus from the $N=2$ algebra one concludes that $O_{\alpha}^{(2)}$ is closed but not exact, while the anti-holomorphic deformation $O_{\bar{\alpha}}^{(2)}=s \bar{Q} \Phi_{\bar{\alpha}}$ is BRS-trivial.

Therefore, the derivatives with respect to the anti-holomorphic moduli of generic physical correlators are equivalent to BRS-trivial insertions, and - in absence of BRS anomalies - should vanish.

Topological sigma models can be coupled to topological gravity to give topological string models [3]. In the conventional treatments, this coupling looks relatively harmless: The BRS operator of the coupled system is taken to be the sum of the BRS operator of the matter model with that of the gravitational sector. The physical operators of the matter system are therefore automatically promoted to elements of the cohomology of the total $s$. Gravity couples to the matter via the term $\int \psi G$ in the action, where $\psi$ is the gravitino and $G$ the matter supercurrent. The world sheet super-reparametrization symmetry is fixed by going to the superconformal gauge, after which the gravitino $\psi$ becomes a differential on the moduli space of Riemann surfaces $\mathcal{M}_{g}$ of given genus $g: \psi=d_{m} h$, where $h$ is the two dimensional complex structure, $d_{m}$ is the differential on $\mathcal{M}_{g}$. Thus the correlators $\left\langle\prod_{\alpha} \int O_{\alpha}^{(2)}\right\rangle_{\text {coupled }}$ of the coupled system are reduced to integrals over $\mathcal{M}_{g}$ of the matter correlators $\left\langle\left[\int \psi G\right]^{6 g-6} \prod_{\alpha} \int O_{\alpha}^{(2)}\right\rangle_{\text {matter }}$. In this framework the insertion of BRS-trivial operators in the correlator produces a total derivative on $\mathcal{M}_{g}$, since $\{s, G\}=T$, where $T$ is the stress-energy tensor, and the insertion of $\int \psi T$ is equivalent to a 
derivative with respect to the Riemann moduli. Thus it is expected that the derivative with respect to an anti-holomorphic modulus of string physical correlators yields the integral over $\mathcal{M}_{g}$ of a total derivative. The computation of Cecotti et al. [4] shows however that this integral does not vanish. This has been therefore interpreted as the signal that the boundary of $\mathcal{M}_{g}$ induces an anomaly of the BRS symmetry.

This interpretation is problematic: The existence of a boundaryless compactification of $\mathcal{M}_{g}$ is crucial to prove the very consistency of string theory in general. It ensures independence of physical correlators of gauge fixing as well as of the choice of the BRS representative for physical states.

In fact we will show that, after coupling topological gravity to the topological B-model, the antiholomorphic deformations become non-trivial elements of the $s$-cohomology. This reconciles the antiholomorphic dependence of topological string correlators with the absence of any BRS anomaly.

Since the action of the B model is $s$-exact the complex structure dependence of the model is completely encoded in the BRS operator. The $s$ of the rigid model is essentially the Dolbeault operator $\bar{\partial}$ on $X$ : This is indeed known to depend holomorphically on the complex structure of $X$. However, as it will be shown in the following, the effect of topological gravity is to deform $s$ by adding to it terms proportional to the superghost $\gamma$ - which act as the anti-Dolbeault operator $\partial$ on $X$ [5]. $\partial$ introduces an explicit anti-holomorphic dependence in $s . \gamma$ is a field which vanishes at the points where zero-form observables are inserted: Therefore the cohomology of the deformed $s$ on the local operators is unchanged and matter observables do remain physical. However when $s$ acts on integrated operators like the action the superghost does not vanish. Thus the anti-holomorphic derivatives of the action pick up a term which is not $s$-trivial.

One can indeed work in a gauge - like the superconformal one - which sets $\gamma$ to zero. With this choice antiholomorphic derivatives of the action do appear to be $s$-trivial. However in this gauge correlators are not globally defined forms on moduli space and contact terms - which introduce the antiholomorphic dependence on the $X$ complex structure - are required to restore gauge invariance [6]. In conclusion, the $s$-triviality of the antiholomorphic derivatives is a mere gauge artifact.

\section{Coupling the B-model to topological gravity}

Let $X$ be a Calabi-Yau complex three-fold, and $J$ a complex structure on it defined by a system of complex coordinates $\left(V^{i}, V^{\bar{\imath}}\right)$. The topological rigid B-model on $X$ is characterized by the following BRS transformations [1]:

$$
\begin{array}{ll}
s V^{i}=0 & s V^{\bar{\imath}}=\Sigma^{\bar{\imath}} \\
s P^{i}=d V^{i} & s \Sigma^{\bar{\imath}}=0 \\
s F^{i}=d P^{i} & s H^{\bar{\imath}}=0
\end{array} \quad s \Delta^{\bar{\imath}}=H^{\bar{\imath}}
$$

$P^{i} \equiv P_{\mu}^{i} d x^{\mu}$ is a fermionic one-form on the world sheet $\Sigma . F^{i} \equiv \frac{1}{2} F_{\mu \nu}^{i} d x^{\mu} d x^{\nu}$ is a bosonic auxiliary field with values in the two-forms on the world sheet. $\Sigma^{\bar{\imath}}, \Delta^{\bar{\imath}}$ are fermionic scalar fields. $H^{\bar{\imath}}$ is a bosonic scalar auxiliary field. $V^{i}$ and $V^{\bar{\imath}}$ are fields of ghost number zero: The ghost number assignment of the other fields in (11) is fixed by the fact that the BRS operator $s$ carries ghost number 1 .

The action $S$ of the B-model is s-trivial, $S=s W$. The standard choice for the gauge fermion $W$ is :

$$
W=\int_{\Sigma} G_{i \bar{\jmath}}\left(F^{i} \Delta^{\bar{\jmath}}+\star P^{i} d V^{\bar{\jmath}}\right),
$$

where $G_{i \bar{\jmath}}$ is a (Ricci-flat) Kähler metric on $X$ adapted to the complex structure $J$. To keep invariance under holomorphic reparametrizations of $X$ explicit at each stage of the computations, it is convenient to modify the BRS operator $s$ in (11) as follows

$$
\begin{array}{ll}
s V^{i}=0 & s V^{\bar{\imath}}=\Sigma^{\bar{\imath}} \\
s P^{i}=d V^{i} & s \Sigma^{\bar{\imath}}=0 \\
s F^{i}=D P^{i}+\frac{1}{2} \Sigma^{\bar{\imath}} P^{k} R_{\bar{\imath} k j}^{i} P^{j} & s H^{\bar{\imath}}=-\Sigma^{\bar{k}} \bar{\Gamma}_{\bar{k} \bar{\jmath}}^{\bar{c}} H^{\bar{\jmath}},
\end{array} \Delta^{\bar{\imath}}=H^{\bar{\imath}}-\Sigma^{\bar{k}} \bar{\Gamma}_{\bar{k} \bar{\jmath}}^{\bar{\imath}} \Delta^{\bar{\jmath}}
$$


where $\Gamma_{j k}^{i}$ and $\bar{\Gamma}_{\bar{\jmath} \bar{k}}^{\bar{\imath}}$ are the components of the Levi-Civita connection relative to $G_{i \bar{\jmath}}$. It is then natural to define the covariant operator $\mathcal{S} \equiv s+\Sigma^{\bar{\imath}} \bar{\Gamma}_{\bar{\imath}}$, where the connection term is understood to act on fields like $\Delta^{\bar{\imath}}$ and $H^{\bar{\imath}}$ - with values in the antiholomorphic tangent of $X . \mathcal{S}$ is nilpotent because the curvature two-form associated to $\Gamma$ is of type $(1,1)$.

The topological rigid B-model can be coupled to topological gravity, whose fields are the world sheet metric $g_{\mu \nu}$, the gravitino $\psi_{\mu \nu}$, the diffeomorphism ghost $c^{\mu}$ and the commuting superghost $\gamma^{\mu}$ of ghost number 2. Their BRS transformation laws are [6]:

$$
\begin{array}{rlrl}
s g_{\mu \nu} & =\psi_{\mu \nu}-\mathcal{L}_{c} g_{\mu \nu} & & s c^{\mu}=\gamma^{\mu}-\frac{1}{2} \mathcal{L}_{c} c^{\mu} \\
s \psi_{\mu \nu} & =\mathcal{L}_{\gamma} g_{\mu \nu}-\mathcal{L}_{c} \psi_{\mu \nu} & s \gamma^{\mu}=-\mathcal{L}_{c} \gamma^{\mu},
\end{array}
$$

where $\mathcal{L}_{c}$ denotes the Lie derivative with respect to the vector field $c^{\mu}$. After coupling to topological gravity, the BRS transformation laws of the matter fields must include an infinitesimal diffeomorphisms with parameter $c$. Since $c$ transforms into $\gamma$, extra terms are required to ensure the nilpotency of $s$. Thus, for example, the BRS transformation rules of $V^{i}$ become

$$
s V^{i}=-\mathcal{L}_{c} V^{i}+\cdots=-i_{c} d V^{i}+\cdots,
$$

where $i_{c}$ is the contraction of a form with the vector field $c$. Hence,

$$
s^{2} V^{i}=0=-\mathcal{L}_{\gamma} V^{i}+s(\cdots),
$$

from which one concludes that

$$
s V^{i}=-\mathcal{L}_{c} V^{i}+i_{\gamma}\left(P^{i}\right),
$$

given that $s P^{i}=d V^{i}+\cdots$. Proceeding in this way one arrives to the BRS rules for the coupled system:

$$
\begin{aligned}
& \mathcal{S} V^{i}=i_{\gamma}\left(P^{i}\right) \\
& \mathcal{S} P^{i}=d V^{i}+i_{\gamma}\left(F^{i}\right) \\
& \mathcal{S} F^{i}=D P^{i}+\frac{1}{2} \Sigma^{\bar{\imath}} P^{k} R_{\bar{\imath} k j}^{i} P^{j} \\
& \mathcal{S} V^{\bar{\imath}}=\Sigma^{\bar{\imath}} \\
& \mathcal{S} \Sigma^{\bar{\imath}}=\mathcal{L}_{\gamma} V^{\bar{\imath}} \\
& \mathcal{S} \Delta^{\bar{\imath}}=H^{\bar{\imath}} \\
& \mathcal{S} H^{\bar{\imath}}=i_{\gamma}\left(D \Delta^{\bar{\imath}}\right)+i_{\gamma}\left(P^{i}\right) \Sigma^{\bar{k}} \bar{R}_{i \bar{k} \bar{\jmath}}^{\bar{\imath}} \Delta^{\bar{\jmath}}
\end{aligned}
$$

To simplify notations, we have modified the definition of the covariant operator $\mathcal{S} \equiv s+\Sigma^{\bar{\imath}} \bar{\Gamma}_{\bar{\imath}}+i_{\gamma}\left(P^{i}\right) \Gamma_{i}+$ $\mathcal{L}_{c}$, to include both the Levi-Civita connection acting on fields with values in the holomorphic tangent of $X$ and the action of world sheet infinitesimal diffeomorphisms with parameter $c$. The nilpotency of the $s$ for the coupled system implies that the square of the covariant $\mathcal{S}$ is:

$$
\mathcal{S}^{2}=\left\{D, i_{\gamma}\right\}+i_{\gamma}\left(P^{i}\right) \Sigma^{\bar{\jmath}} R_{i \bar{\jmath}}
$$

The r.h.s. of (10) is the curvature of the infinite-dimensional bundle in field space which underlies the geometry of the system: The total space of this bundle is the product of the space of complex structures of Riemann surfaces $\Sigma$ and the tangent space to the space of maps from $\Sigma$ into $X$. Its structure group is the product of the group of diffeomorphisms of $\Sigma$ and the group of holomorphic reparametrizations of $X$.

\section{Dependence of $s$ on the complex structure of $X$}

The action of the B-model is $s$-trivial. Therefore the physical correlators are independent of the choice of the Kähler metric $G_{i \jmath}$ which appears in Eq. (2). However the definition of the BRS operator $s$ requires choosing a complex structure $J$ on $X$ : Physical correlators will be therefore functions of $J$. 
Because of the triviality of the action, the functional measure for physical correlators localizes on the field zero modes. In particular, when discussing operators depending on world sheet scalars - such as $V^{i}, V^{\bar{\imath}}, \Sigma^{\bar{\imath}}, \Delta^{\bar{\imath}}$ - we can assume that the fields are constant.

Before coupling to topological gravity, $s$ acts on functions of $V^{i}, V^{\bar{\imath}}, \Sigma^{\bar{\imath}}$ as the Dolbeault differential, since

$$
s O\left(V^{i}, V^{\bar{\imath}}, \Sigma^{\bar{\imath}}\right)=\Sigma^{\bar{\imath}} \partial_{V^{\bar{\imath}}} O\left(V^{i}, V^{\bar{\imath}}, \Sigma^{\bar{\imath}}\right),
$$

Thus, to each element $\omega_{\bar{\imath}_{1} \ldots \bar{\tau}_{q}}(V, \bar{V}) d V^{\bar{\imath}_{1}} \ldots d V^{\bar{\imath}_{q}}$ of the $H^{(0, q)}(X)$ Dolbeault cohomology of forms on $X$ of type $(0, q)$, there corresponds an observable $\omega_{\bar{\imath}_{1} \ldots \bar{z}_{q}}(V, \bar{V}) \Sigma^{\bar{\imath}_{1}} \ldots \Sigma^{\bar{\imath}_{q}}$ of ghost number $q$.

Moreover

$$
s\left(G_{i \bar{\jmath}} \Delta^{\bar{\jmath}}\right)=G_{i \bar{\jmath}} H^{\bar{\jmath}} \equiv H_{i}
$$

$H_{i}$ appears in the action as an auxiliary field and the equation of motion relative to $F^{i}$ gives: $H_{i}=$ $G_{i \bar{\jmath}} i_{\gamma}\left(\star d V^{\bar{\jmath}}\right)$. Thus, as long as one considers local operators which do not involve $F^{i}$, it is legitimate to take $s \Delta_{i}=0$, with $\Delta_{i} \equiv G_{i \bar{\jmath}} \Delta^{\bar{\jmath}}$. It follows that $\omega_{\bar{\jmath}_{1} \ldots \bar{\jmath}_{q}}^{i_{1}}(V, \bar{V}) \Delta_{i_{1}} \ldots \Delta_{i_{p}} \Sigma^{\bar{c}_{1}} \ldots \Sigma^{\bar{\nu}_{q}}$ is an element of the cohomology of $s$ if $\omega_{\bar{\jmath}_{1} \ldots \bar{j}_{q}}^{i_{1} \ldots i_{p}}(V, \bar{V}) \partial_{V^{i_{1}}} \ldots \partial_{V^{i_{p}}} d V^{\bar{i}_{1}} \ldots d V^{\bar{z}_{q}}$ is an element of the cohomology $H^{(0, q)}\left(X, \Lambda^{p} T^{(1,0)} X\right)$ of $(0, q)$ forms with values in the bundle $\Lambda^{p} T^{(1,0)}$, the $p^{\text {th }}$ exterior power of the holomorphic tangent bundle $T^{(1,0)} X$.

Among the observables of this class, those relative to elements of $H^{(0,1)}\left(X, T^{(1,0)} X\right)$ correspond to holomorphic deformations of the complex structure of $X$. Let us choose a basis for them

$$
O_{\alpha}=\Delta_{i} \mu_{\alpha \bar{\jmath}}^{i} \Sigma^{\bar{j}} \equiv \Delta^{\mathrm{t}} \mu_{\alpha} \bar{\Sigma}
$$

where $\alpha=1, \ldots, \operatorname{dim} H^{(0,1)}\left(X, T^{(1,0)} X\right)$ and we introduced an obvious matrix notation for $\mu_{\alpha \bar{\jmath}}^{i}, \Delta_{i}$ and $\Sigma^{\bar{j}}$ and $\mathrm{t}$ denotes the transpose.

An explicit construction of $\mu_{\alpha \bar{\jmath}}^{i}$ makes use of the Beltrami parametrization for $J$ given by the equations:

$$
d V=\Lambda(d v+\mu d \bar{v}) \quad d \bar{V}=\bar{\Lambda}(d \bar{v}+\bar{\mu} d v)
$$

where $(v, \bar{v}) \equiv\left(v^{i}, v^{\bar{\imath}}\right)$ is a fixed system of complex coordinates and $\mu \equiv \mu_{\bar{\jmath}}^{i}, \bar{\mu} \equiv \bar{\mu}_{j}^{\bar{\imath}}$ are the Beltrami differentials. A natural complex structure on $\mathcal{M}_{J}$, the moduli space of complex structures on $X$, is defined by declaring $\mu(\bar{\mu})$ to be a (anti)holomorphic function of $J$. Let $\left(m_{\alpha}, m_{\bar{\alpha}}\right)$ be complex coordinates on $\mathcal{M}_{J}$. The holomorphic derivative of $d V$ with respect to $m_{\alpha}$ is a form of mixed type [7]:

$$
\partial_{\alpha} d V=A_{\alpha} d V+\mu_{\alpha} d \bar{V}
$$

Since $\mu_{\alpha} d \bar{V}=\bar{\partial} \partial_{\alpha} V$, it follows that $\mu_{\alpha} d \bar{V}$ is indeed an element of $H^{(0,1)}\left(X, T^{(1,0)} X\right)$. By plugging Eq. (14) into Eq. (15), one can write $A_{\alpha}$ and $\mu_{\alpha}$ in terms of the Beltrami differentials:

$$
\begin{aligned}
A_{\alpha} & =\partial_{\alpha}\left(\Lambda A^{-1}\right) A \Lambda^{-1} \\
\mu_{\alpha} & =\Lambda \partial_{\alpha} \mu \bar{A} \bar{\Lambda}^{-1}
\end{aligned}
$$

where $A \equiv(1-\mu \bar{\mu})^{-1}$.

Let us use the Beltrami parametrization to exhibit the dependence of the Dolbeault operator $\bar{\partial}_{J} \equiv$ $d V^{\bar{\imath}} \partial_{V^{\bar{\imath}}}$ on $J$ :

$$
\bar{\partial}_{J}=(d \bar{v}+\bar{\mu} d v)^{\mathrm{t}} \bar{A}^{\mathrm{t}}\left(\partial_{\bar{v}}-\mu^{\mathrm{t}} \partial_{v}\right) \equiv \overline{\mathrm{e}}^{\mathrm{t}}\left(\partial_{\bar{v}}-\mu^{\mathrm{t}} \partial_{v}\right)
$$

where $\mathrm{e}^{\bar{\imath}}=[\bar{A}(d \bar{v}+\bar{\mu} d v)]^{\bar{v}}$ is a basis for the one-forms of type $(0,1)$ in the complex structure $J$. This redefinition of the basis of one-forms of type $(0,1)$ renders $\bar{\partial}_{J}$ manifestly holomorphic in $J$.

Thus, working in this basis, the elements of the Dolbeault cohomology - i.e. the solutions of $\bar{\partial}_{J} \omega=0$ - can be taken to depend holomorphically on $J$. This means that the vector bundle over $\mathcal{M}_{J}$ whose fiber is $H^{(0, q)}(X)$ is a holomorphic vector bundle. The same is true for the vector bundle with fiber $H^{(0, q)}\left(X, \Lambda^{p} T^{(1,0)} X\right)$. 
This implies that the BRS operator for the B-model before coupling to topological gravity does not depend on $\bar{\mu}$, as long as one makes the redefinition

$$
\bar{\Sigma}=\bar{\Lambda} \bar{A}^{-1} \bar{\sigma}
$$

in the anti-holomorphic sector. Furthermore the action of the BRS operator on the new fields should not depend on the non-local integrating factor $\Lambda$. It is easy to verify that this dictates the following redefinitions in the holomorphic sector:

$$
\Delta^{\mathrm{t}}=\delta^{\mathrm{t}} \Lambda^{-1} \quad H^{\mathrm{t}}=h^{\mathrm{t}} \Lambda^{-1} \quad P=\Lambda \rho \quad F=\Lambda f
$$

In this field basis, the observables (13) become:

$$
O_{\alpha}=\delta^{\mathrm{t}} \partial_{\alpha} \mu \bar{\sigma}
$$

which shows explicitely that in the new field basis one can choose a set of observables which are holomorphic in $J$.

After coupling to topological gravity the action of $s$ is modified to be:

$$
\begin{aligned}
s & =\Sigma^{\bar{\imath}} \partial_{V^{\bar{\imath}}}+i_{\gamma}\left(P^{i}\right) \partial_{V^{i}}+\ldots \\
& =\bar{\sigma}^{\mathrm{t}}\left(\partial_{\bar{v}}-\mu^{\mathrm{t}} \partial_{v}\right)+i_{\gamma}(A \rho)^{\mathrm{t}}\left(\partial_{v}-\bar{\mu}^{\mathrm{t}} \partial_{\bar{v}}\right)+\ldots,
\end{aligned}
$$

and $\mathcal{S} \bar{\Sigma}=\mathcal{L}_{\gamma} \bar{V}, \mathcal{S} i_{\gamma}(P)=\mathcal{L}_{\gamma} V$

The ghost $c$ and the superghost $\gamma$ vanish on the points of the world sheet where local zero-forms observables are inserted. Thus $s$ reduces on such observables to the $s$ of the rigid theory. In particular, the operators (13) remain physical after coupling to topological gravity.

However, on operators integrated over the world sheet, such as the action, the $\gamma$ dependent term in Eq. (21) does not vanish: It induces a dependence of $s$ on $\bar{\mu}$. This is the origin of the holomorphic anomaly of the topological string B model.

Let us evaluate the dependence of $s$ on $\bar{\mu}$ :

$$
I_{\bar{\alpha}} \equiv\left[\partial_{\bar{\alpha}}, \mathcal{S}\right]=i_{\gamma}\left(P^{i}\right)\left[\partial_{\bar{\alpha}}, \partial_{V^{i}}+\ldots\right]=-i_{\gamma}\left(P^{i}\right) \mu_{\bar{\alpha} i}^{\bar{j}} \partial_{V^{\bar{j}}}+\ldots
$$

This gives for the antiholomorphic dependence of the action the following result:

$$
\partial_{\bar{\alpha}} S=I_{\bar{\alpha}} W+s\left(\partial_{\bar{\alpha}} W\right)=\int_{\Sigma} G_{i \bar{\jmath}} D \star P^{i} \mu_{\bar{\alpha} k}^{\bar{j}} i_{\gamma}\left(P^{k}\right)+s\left(\partial_{\bar{\alpha}} W\right),
$$

from which one derives the Ward identity:

$$
\partial_{\bar{\alpha}}\left\langle\prod_{\alpha} O_{\alpha}\right\rangle=\left\langle\int_{\Sigma} G_{i \bar{\jmath}} D \star P^{i} \mu_{\bar{\alpha} k}^{\bar{j}} i_{\gamma}\left(P^{k}\right) \prod_{\alpha} O_{\alpha}\right\rangle .
$$

Let us end by remarking that $I_{\bar{\alpha}} W$ is BRS-closed modulo the equation of motions since

$$
s I_{\bar{\alpha}} W=-I_{\bar{\alpha}} s W=-I_{\bar{\alpha}} S,
$$

which ensures that the correlator in the r.h.s. of Eq. (24) is gauge-invariant.

\section{References}

[1] E. Witten, "Mirror Manifolds and Topological Field Theory", in Essays on Mirror Manifolds, edited by S. Yau, International Press (Honk Kong 1992).

[2] S. Cecotti and C. Vafa, Nucl. Phys. B367 (1991) 359. 
[3] R. Dijkgraaf, E. Verlinde and H. Verlinde, "Notes on Topological String Theory and 2-D Quantum Gravity", in String Theory and Quantum Gravity '90, proceedings of the Trieste Spring School and Workshop, ICTP, Trieste, 1990, World Scientific, (Singapore 1991).

E. Witten, Nucl. Phys B340 (1990) 281.

[4] M. Bershadsky, S. Cecotti, H. Ooguri and C. Vafa, Commun. Math. Phys. 165 (1994) 311.

[5] C.M. Becchi, S. Giusto and C. Imbimbo, "The BRST structure of twisted $N=2$ algebra", forthcoming in the proceedings of the International Conference on Secondary Calculus and Cohomological Physics, Moscow, 24-31 August 1997, Contemporary Mathematics series of the American Mathematical Society.

[6] C.M. Becchi and C. Imbimbo, Nucl. Phys. B462 (1996) 571.

[7] P. Candelas and X. de la Ossa, Nucl.Phys. B355 (1991) 455. 